\title{
Ruled Based Construction and the Materialization of a Line in Motion
}

OLIVIER OTTEVAERE

The University of Hong Kong

From the work of the Architect and Engineer Felix Candela on Hyperbolic Paraboloid surfaces for thin shell construction, the paper retraces how ruled geometries, generalized by a sequence of rotating lines, directly regulate procedures of construction for concrete formwork, made of straight timber elements.

From the conception of three timber structures, The Pinch, Sweep and Warp, it is discussed how such geometrical principles are further explored structurally, materially and spatially. Finally, a series of subsequent physical experiments are put forward in search of new shell topologies, generated solely from a moving line described by time and movement. For the timber structures, sequences of changing wooden trusses capture the movement of a line to support ruled decks performing as new active grounds. The results are a series of three small scale social programs: a library, a play area and a roadside marketplace.

They were each built with students and with the help of a local timber workshop, developing construction methods for adapting highly articulated geometries to simple traditional techniques.

Situated at the intersection of teaching and research, experimentation and on-site construction, complex geometry and local craftsmanship, these design-build projects engage with full scale construction in difficult sites through experiential learning.

\section{INTRODUCTION}

The Pinch, Sweep and Warp (Figure 1a, 1b, 1c) are three earthquake reconstruction projects located in Yunnan Province, China. Partly built with students of architecture from The University of Hong Kong and
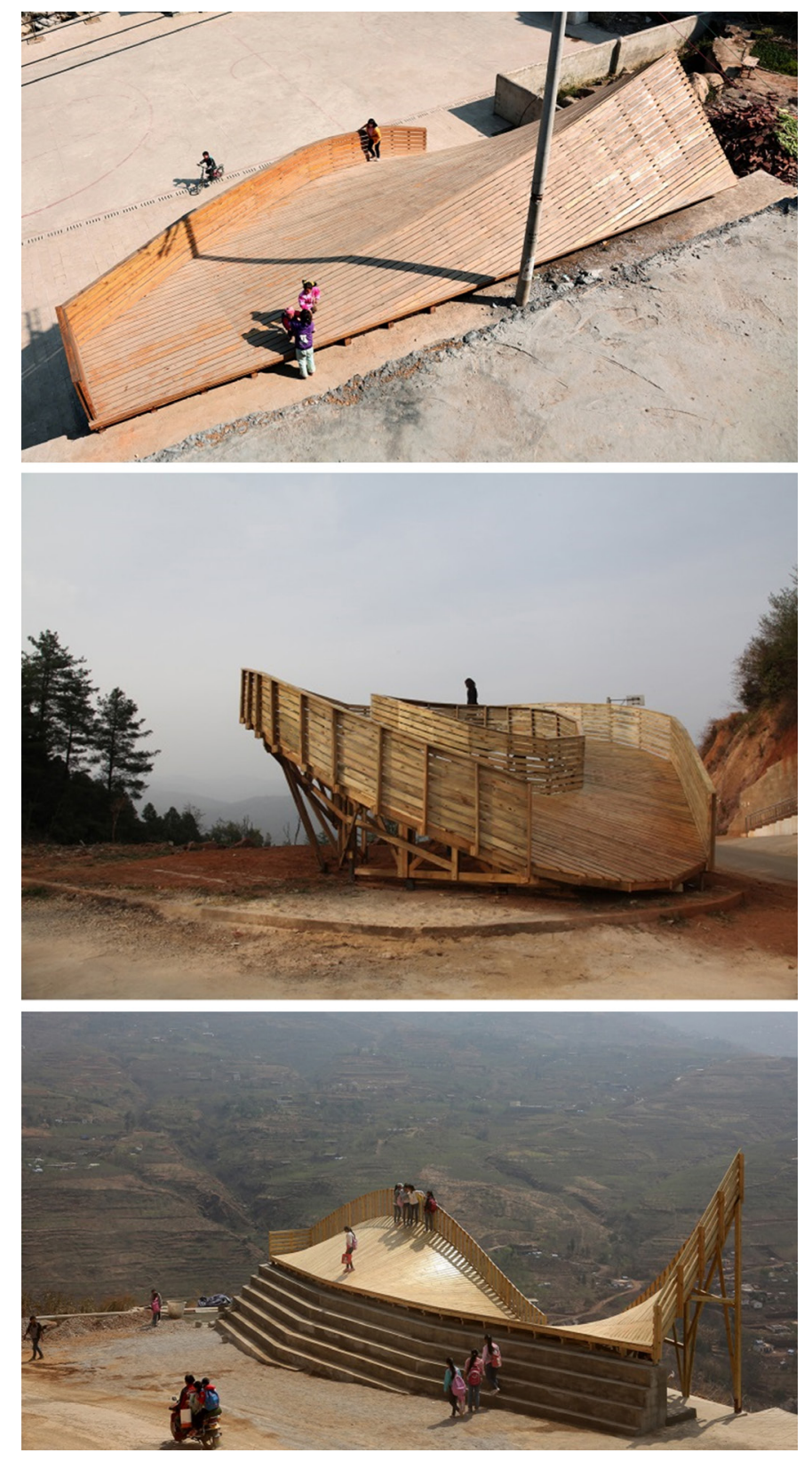

Figure 1: Pinch, Sweep, Warp. 

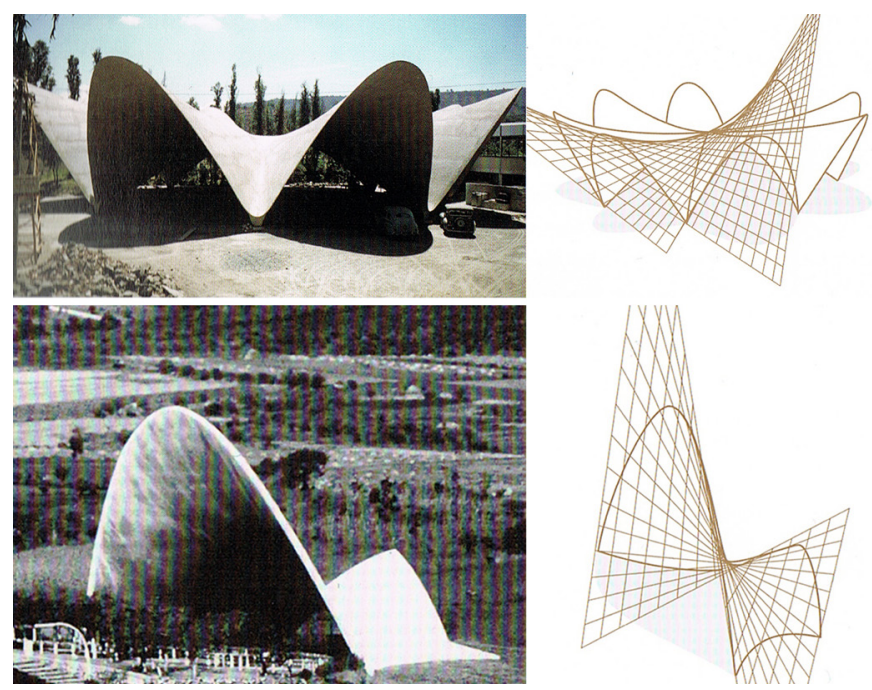

Figure 2a: Los Manantiales Restaurant, Mexico City, 1958 by Felix Candela and straight-line generators drawing

Figure 2b: Chapel Lomas de Cuernavaca, 1958 by Felix Candela and straight line generators drawing

with the help of a local timber workshop, these projects maximize the social and programmatic impact of small scale interventions. Located in a remote mountainous landscape of peaks and valleys, each project was designed with a strategy of maximizing the use and experience of the landscape via structural wooden trusses and decked, ruled surfaces. The results are three structures, each with a diversity of social programs.

\section{HISTORICAL BACKGROUND}

Precedents for the construction of the three structures include Felix Candela's work on thin shell structures. The rationalization of his hyperbolic paraboloid (double curvature) into sequences of rotating straight-line generators (Garlock and Billington 2008) enabled him to conceive falseworks of great economy. His translation of hypars into linear geometry facilitated the construction of formworks made from straight timber planking.

Although labor intensive, complex shells such as the Los Manantiales Restaurant or the Chapel Lomas de Cuernavaca (Figure 2a, 2bvv), were still made possible with low-skill workers and with the availability of cheap labor in Mexico at the time.

The Pinch, Sweep and Warp are direct experiments on the conversion of doubly curved surfaces to linear geometry and to material constraints, in rural China. Each project is specifically demarcated by a sequence of changing wooden trusses. The trusses are organized transversally for the Pinch, tangentially for the Sweep and longitudinally for the Warp. A line in motion is arrested by the succession of trusses to transform a ruled deck into a new active ground (Figure 3 ).

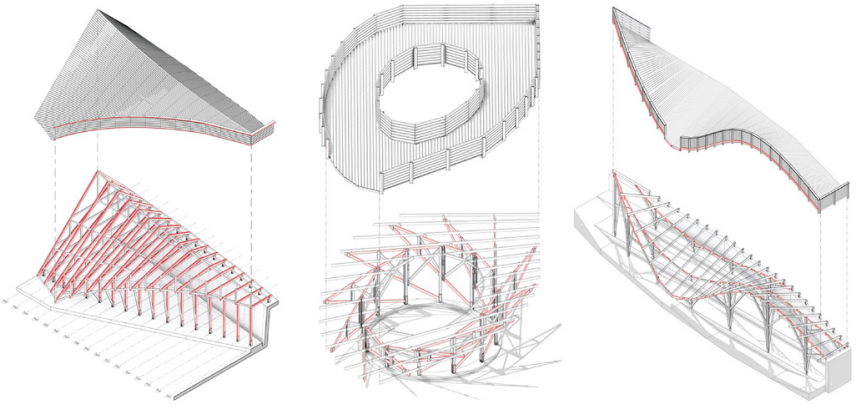

Figure 3: Catalogue of trusses for Pinch, Sweep and Warp.

\section{THE PINCH}

Context

The Pinch is a library and community center of around 80 sqm in Shuanghe Village, Yunnan Province, China. The project is part of a government led reconstruction effort after an earthquake in September 2012. The majority of village houses were destroyed, leaving the residents living in tents for up to one year. Though the government sponsored new houses and a large central plaza, it remained an empty site. The University of Hong Kong decided to sponsor the design of a new library building that might serve to activate the community. Due to limited funds, the building was designed as a two phase project.

\section{Construction}

The primary structure concluded phase one while phase two concentrated on the enclosure and furnishing. The main structure consists of a series of 17 timber trusses arranged in parallel. They evolve from a small door at one end to a 6 meter high pitched space at the other, housing the library below.

Each truss sets against an existing 4 meter high retaining wall for structural support and earthquake resistance. The design strategy involved a series of trusses spanning the level difference, acting as a bridge between the rebuilt village and the new memorial plaza. The section of each truss changes to create both a gradual incline (to bring people down) and then a sharp upward pitch (to elevate the roof). The trusses were covered in an aluminium waterproofing layer and timber decking.

On the interior, the trusses extend downward to support a floating bookshelf. Simple traditional school benches are used as chairs. The polycarbonate doors can open to create a completely open space extending out to the plaza.

All trusses were prefabricated flat on site. The original $2 \mathrm{~d}$ drawings were redrawn with handmade compasses and straightedges by local carpenter at 1 to 1 scale directly on the floor of the plaza. The various truss elements were then placed on top of the 1 to 1 drawing, cut to sizes and assembled to complete each truss (Figure 4). Projective 


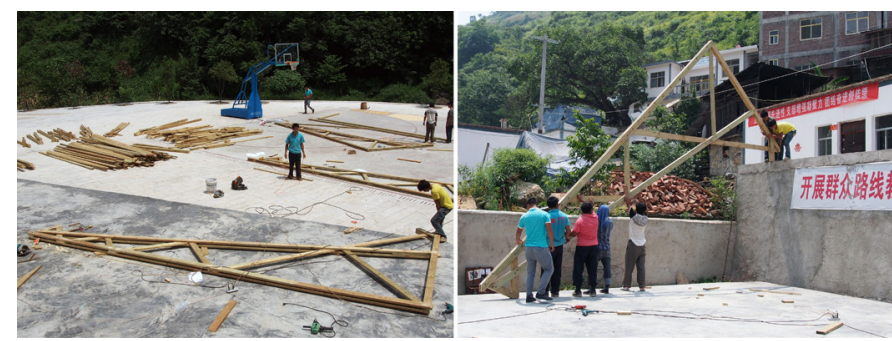

Figure 4: Prefabrication and installation of trusses on site

Geometry was the mean by which the primary structure was efficiently erected. The process involved translating the three-dimensional geometry into local construction techniques and procedures from a single timber section: $11 \mathrm{~cm}$ wide by $3.3 \mathrm{~cm}$ thick.

Once in place, the 17 trusses served as the main guide for the completion of the decking. The top surface was decked in parallel planks running along the uppermost ridge.

Due to the deck's three- dimensionality, the timber planks needed to perform a certain amount of bending and torqueing. To assess the bending limit of the material, initial physical tests were conducted. The established material properties consequently informed the calibration of the overall geometry. The trusses alone condition the space of the library below, best expressed from the changing depth of its ceiling plane.

The overall process resulted in the development of a surprisingly diverse form through simple means. As a single structure it is simultaneously a bridge, seating and play area, roof and bookshelf (Figure 5). The project is juxtaposition of above and below, simple and complex, contemporary forms achieved through local means and techniques.

Since completion, The Pinch has been adopted by the villagers as a monument for the earthquake rebuilding.

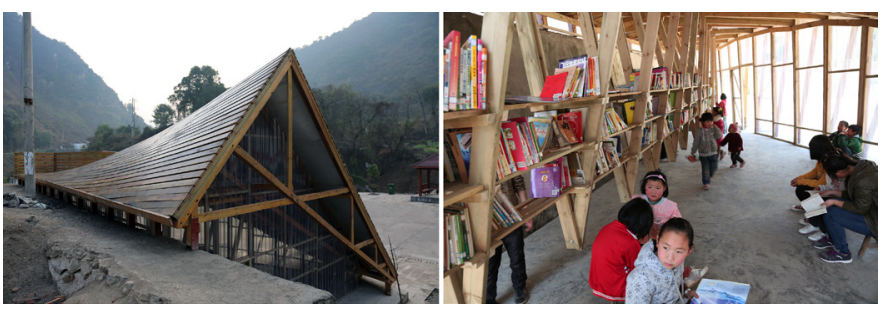

Figure 5: The Pinch, above and below

One of the advantages of small projects is the possibility for experimentation. The Pinch was the first in a series of timber structures. The second and third structure (Sweep and Warp), make use of similar construction procedures, yet of different structural arrangements and spatial organizations. They also involve students' participation.

\section{THE SWEEP}

Context

The Sweep is a viewing platform and play area of about $60 \mathrm{sqm}$, built in a Bai minority village in Yunnan Province. The project is located at the entrance to the local primary school. Students live in the school on weekdays and are picke $d$ up by parents for the weekend. The platform is a natural waiting area for parents and students, offering a shaded space below.

Though the plan arrangement of trusses is simple, the overall structure delivers a diversity of views and spaces, constructing a 360 degree panorama of village houses, mountains, valley, and farmland. Conducted as an experiential learning project in the Department of Architecture at The University of Hong Kong, the project was built in 6 days with 65 students.

\section{Construction}

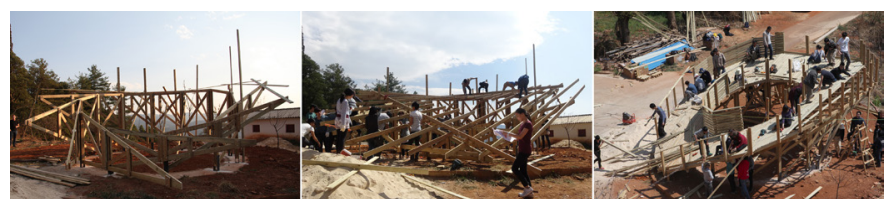

Figure 6: fabrication of trusses, ruled beams and ruled deck

The timber structure is designed as a series of 12 different trusses arranged tangentially into a self-reinforced ring. Stiffness of the ring is ensured by connecting every truss to its front and back neighbours. Straight beams laid parallel are sandwiched between the trusses below and wooden boards above, running in the opposite direction to form the decking (Figure 6).

Each triangular truss cantilevers up to 4 meters to support a decked surface. As the trusses descend and ascend off the edge of a cliff, the ruled surface evolves from the ground upward, culminating in a spectacular view across a valley of rice terraces.

The same contractor team oversaw the overall construction and assisted students with the assembly of the structure. Equipped with sets of drawings, they actively engage in smaller groups with local carpenters in the building of the project. These design-build projects embolden students with full scale construction exposure in complex sites. Their feedback noted the experience intense, yet pedagogically much enriching. The project has since become part of the primary school playground (Figure 7).
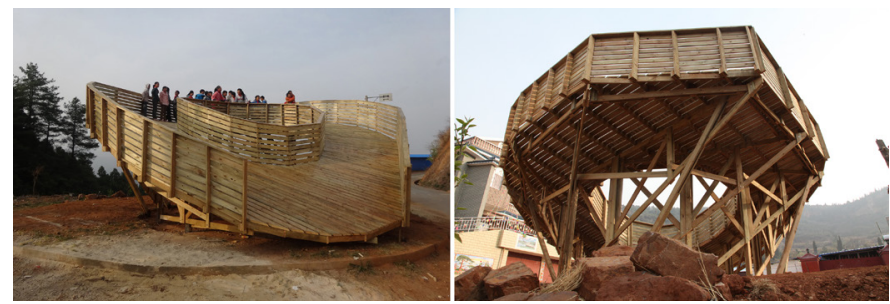

Figure 7: The Sweep above and below 


\section{THE WARP}

Context

The Warp is a rest area and roadside market of 130sqm, also built as part of a post-earthquake reconstruction in Ludian town, Yunnan, China. Situated in an ethnically diverse region with a Muslim majority population, the project serves as a meeting point and look out along the main entry road to a mountain village. The project provides three key spaces, stepped concrete seating area for selling fruit and produce, a wooden deck for viewing and a covered resting and eating area below. Formally, the deck extends toward the scenic valley, transforming from a straight line into a sine curve. Its peaks and valleys mimic the landscape while providing two mirrored spaces (above and below) for viewing and resting.

\section{Construction}

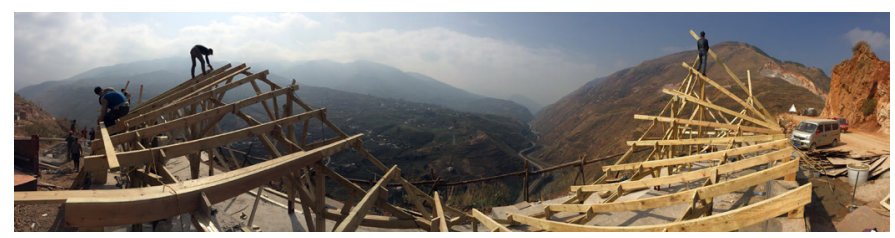

Figure 8: The Warp under construction

The principal elements are the same than Pinch and Sweep; prefabricated timber trusses, supporting a deck, described in linear or ruled geometry. In this instance, 17 columns, placed longitudinally, are fanning upwards to delineate three long sinusoidal profiles onto which a series of straight beams are resting transversally (Figure 8).

From left to right, each straight beam rotates upwards to a viewing peak, then downwards to meet the ground and upwards again towards the extensive landscape. Rows of spacers link the sequence of rotating beams to receive the decking, also made of linear timber planks.

Although made of the same material and construction logic, the three projects experiment with distinctive structural principles via different arrangements of trusses.

Consequently, each spatial experience is rendered unique by its ruled deck, expanding and contracting as ceiling and new ground (Figure 9). While moving and pausing onto these new tectonic tops, the participant is made more receptive of the all-encompassing intensity of the Yunnan terrain.

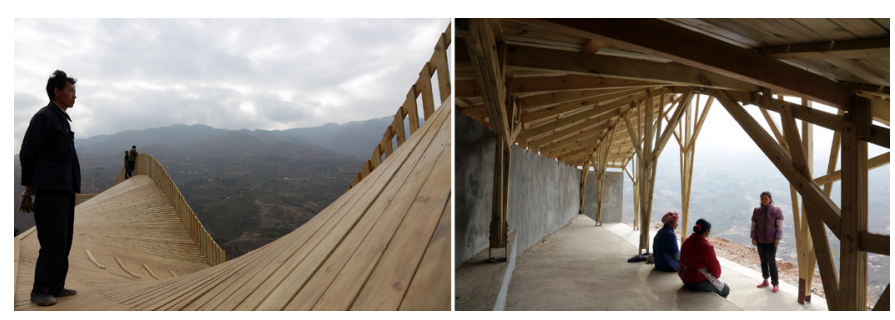

Figure 9: The Warp above and below

\section{CONCLUSION}

These projects engage the reconstruction processes led by the government, filling in the gap between infrastructure and house reconstruction. They instead focus on how a single small structure can address a maximum of community needs. The projects are the result of on-going collaboration with a local timber workshop, developing techniques for adapting complex geometries to simple traditional techniques. Throughout the reconstruction process, many traditional wooden buildings are abandoned in favor of newer concrete frame and brick infill structures. However, these projects revitalize the role of local timber craft, through strategies of responsive, programmatically diverse and economical design. Collectively the series explores the activities of buying and selling, bridging, resting, viewing, eating, reading, and playing. In each of these sites, the project has become a monument to the rebuilding effort.

Ultimately the project is an independent exploration of the intersection between teaching and research, experimentation and onsite construction, contemporary design methods and local craftsmanship. Both multifaceted and site specific, Pinch, Sweep, Warp is a project that is both prototypical and site specific architectural response.

Over the course of 3 years, the projects bring together the efforts of government, charity and the university. By defining an alternate possibility to fund and build architecture, the project emphasizes economy of means with maximum impact, balancing community use with strong spatial experience, and most importantly rebuilding a community by demonstrating value through design. As an architectural effort, its main aspiration is to educate.

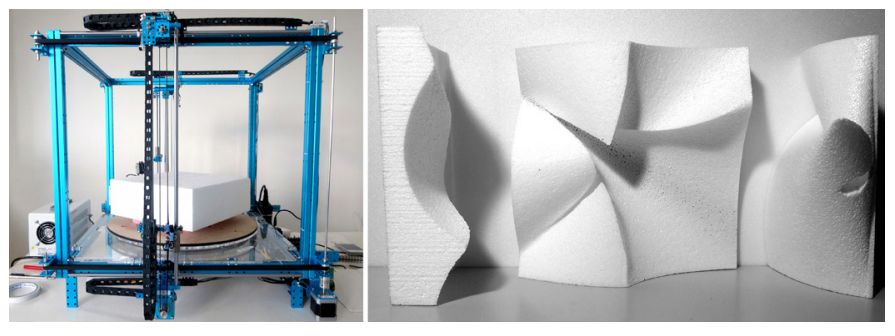

Figure 10: 5-axis automated hot wire and EPS foam mould

\section{LINE ON FIRE}

This last section further speculates on ruled based geometries by describing new continuous surfaces generated from the motion of a straight line. A custom-made automated 5-axis hot wire is utilized as the main research tool (Figure 10).

By inputting specific protocols for synchronized motions (4 translations and 1 rotation), new topologies emerge defined by movement and time. Pairs of $2 \mathrm{~d}$ profiles are inputted from 4 automated axes. The hot wire cutter then takes care of deriving the resulting ruled geometry linking the different sections. This procedure is further employed to section EPS foam blocks into part-moulds for thin shell concrete casting (Figure 10). Taxonomies of EPS-plugs subsequently make up for the negative volumes in between which the concrete is then poured.

In addition to movement, a time factor was introduced in subsequent experiments. This is implemented through the 5 th rotational axis. 
New slab topologies arise from incremental protocols on a line moving in space at irregular intervals. Although the project is still in its infancy, early findings present unique slab topologies, whose forms would be difficult to preconceive through other means of digital fabrication.

By virtue of being described by a succession of straight lines, these intricate slabs retain an efficient and a direct link to timber formwork (made of straight planks) and to large-scale construction.
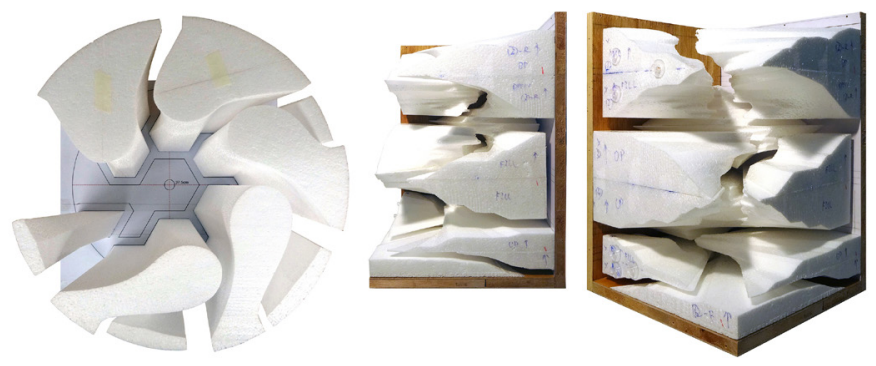

Figure 11: ruled-based formwork experiments for concrete casting

As much prominence in (digital) fabrication has been placed on surface definition machined from sheet materials, the presented prototyping technique seek to reinstate the merits of working with volumetric materials. To this effect, it resonates with an effective method invented by Philippe De L'Orme, now called stereotomy. This art of stone carving made efficient use of projective geometry by translating spatially complex solids into two-dimensional templates to guide the stone cutter in the carving of a block (Evans 1995). Although this time, by operating internally in the slicing of a block (Figure 11) from the automation of a line moving in space, new spatial articulations emerge from active geometries solely defined by time and movement.

\section{ENDNOTES}

1. (Book) M. Garlock and D. Billington, 'Felix Candela, Engineer, Builder, Structural Artist', (New Haven, Yale University Press, 2008), p.148.

2. (Book) R. Evans, 'Drawn Stone'. The Projective Cast, Architecture and its Three Geometries, (Cambridge, The MIT Press, 1995), pp. 179-192. 